\title{
Autoeficacia Cuestionario de Autorregulación de Estudio Readiness
}

\author{
Fabiola Sáez Delgado¹, Claudio Bustos Navarrete, Alejandro Díaz Mujica \\ Universidad de Concepción, Concepción, Chile
}

\section{RESUMEN}

La investigación ha demostrado la importancia de los instrumentos que miden la autoeficacia ligada a áreas de funcionamiento diferenciado, como el aprendizaje autorregulado. El objetivo de este trabajo fue proyectar y estudiar el comportamiento psicométrico del Cuestionario de Autoeficacia para la Autorregulación de la Disposición al Estudio. Se aplicó el instrumento, compuesto por nueve ítems, a una muestra por conveniencia de 695 estudiantes, en cinco universidades de la provincia de Concepción, CHILE. Mediante análisis factorial exploratorio, fue encontrada una estructura con un factor general $(M=3.87 ; D E=0.65)$ y tres sub-factores, que fue demostrada en un análisis factorial confirmatorio. El instrumento muestra niveles adecuados de confiabilidad $(\alpha=0.84)$, una estructura latente con buen ajuste para los datos y correlaciones leves, pero estadísticamente significativas, con clasificaciones autoreferidas. Por lo tanto, el instrumento presenta propiedades psicométricas adecuadas para ser aplicadas en formato de auto-informe a distintos grupos de estudiantes.

Palabras-clave: autoeficacia; autorregulación; estudiantes universitarios; análisis factorial.

\section{RESUMO - Questionário de Autoeficácia para a Autorregulação para Estudar (Readiness)}

The investigation has demonstrated the importance of instruments that measure self-efficacy linked to areas of differential functioning, such as self-directed learning. The objective of this work is to design and study the psychometric behavior of the SelfEfficacy Questionnaire for Self-Directed-Learning. The instrument, composed of nine items, was applied to a convenience sample of 695 students from five universities in the province of Concepción, Chile. Through exploratory factor analysis, a structure with one general factor $(M=3.87, S D=0.65)$ and three sub-factors was found, which was proved through confirmatory factor analysis. The instrument shows adequate levels of reliability $(\alpha=0.84)$, a latent structure with good fit for the data, and slight but statistically significant correlations with self-reported classifications. Therefore, the instrument has adequate psychometric properties to be applied in a self-report format for different groups of students.

Keywords: self-efficacy; self-regulation; university students; factor analysis.

\section{ABSTRACT - Self-Efficacy Questionnaire of the Self-Directed-Learning Readiness}

A investigação tem demonstrado a importância dos instrumentos que medem a autoeficácia ligada às áreas de funcionamento diferencial, como a aprendizagem autorregulada. O objetivo deste trabalho é projetar e estudar o comportamento psicométrico do Questionário de Autoeficácia para a Autorregulação da Disposição para Estudar. O instrumento, composto por nove itens, foi aplicado em uma amostra por conveniência de 695 estudantes de cinco universidades da Província de Concepción, Chile. Por meio de análise fatorial exploratória, foi encontrada uma estrutura com um fator geral $(M=3,87 ; D E=0,65)$ e três subfatores, a qual foi provada em uma análise fatorial confirmatória. O instrumento mostra níveis adequados de confiabilidade $(\alpha=0,84)$, uma estrutura latente com bom ajuste para os dados e as correlações leves, mas estatisticamente significativas, com classificações autorreferidas. Portanto, o instrumento apresenta propriedades psicométricas adequadas para serem aplicadas em formato de autorrelato, para diferentes grupos de alunos.

Palavras-chave: autoeficácia; autorregulação; estudantes universitários; análise fatorial.

Desde la perspectiva social cognitiva, el aprendizaje no es un rasgo personal que los estudiantes poseen o no poseen, sino que comprende el uso selectivo de procesos específicos o estrategias para cada tarea de aprendizaje, procesodenominado autorregulación del aprendizaje.En la literatura existen diferentes definiciones del concepto de autorregulación del aprendizaje. Todas coinciden en entenderlo como un proceso activo, crítico, y reflexivo, donde el estudiante dirige su propia actuación, lo que contribuye a que el individuo alcance su desarrollo pleno, 
y con un sentido de autodeterminación del aprendizaje (Zimmerman, 2008).

La autorregulación del estudio es parte del proceso más amplio, de la autorregulación del aprendizaje (Almeida \& Salcedo, 2013). El concepto de autorregulación del estudio se entiende como una actividad constante en la vida de los estudiantes, en la cuales éstos deben decidir qué hacer mientras estudian sin la guía de un profesor (Soderstrom \& Bjork, 2014). El estudio autorregulado permite a los estudiantes tomar decisiones al momento de enfrentar una tarea, como cuánto tiempo se debe dedicar al estudio, las cuales descansan en un monitoreo preciso de aprendizaje continuo, y al uso apropiado de estrategias de disposición al estudio (Kornell \& Bjork, 2007).

El modelo de autorregulación de Zimmerman (2002) considera tres fases en el proceso de autorregulación -planificación, ejecución y evaluación-, cada una de las cuales se asocia a un tipo de estrategia de autorregulación: (a) estrategias de disposición al estudio, utilizadas en la fase de planificación, (b) estrategias cognitivas, correspondiente a la fase de ejecución y (c) estrategias metacognitivas, asociadas fundamentalmente a la fase de evaluación (Pérez, Valenzuela, Díaz, González-Pienda, \& Núñez, 2011; Zimmerman, Kitsantas, \& Campillo, 2005).

El modelo PLEJE (Rosário et al., 2007) complementa el modelo cíclico de Zimmerman (2002). Plantea que cada fase del modelo de Zimmerman posee a su vez subfases de planificación, ejecución y evaluación (PLEJE) de las actividades en el proceso de aprender. Esto implica por tanto, que en la fase de planificación existen acciones de preparación, de ejecución y de autoevaluación, generándose un subciclo de autorregulación de este proceso, que ayuda al estudiante a desarrollar ajustes de acuerdo al resultado.

En la fase de planificación, los estudiantes recurren a diversas estrategias de disposición al estudio que incluyen: 1. establecimiento de objetivos académicos, 2. gestión del tiempo de académico, 3. organización de recursos materiales y ambientales y 4 . disposiciones motivacionales (Elvira-Valdés \& Pujol, 2014; Pérez et al., 2011; Zimmerman, 2008). Este tipo de estrategias mejora las condiciones en que se produce el aprendizaje (Zimmerman, 2008). Posterior a esto, se produce la reacción y reflexión sobre el esfuerzo y el tiempo empleado gastado en la tarea, lo cual constituye un importante aspecto para cambiar la conducta en situaciones futuras (Yan, Thai, \& Bjork, 2014).

\section{Autoeficacia para la autorregulación de la disposición al estudio}

la autoeficacia se relaciona con las creencias de las personas en su capacidad para producir determinados logros (Bandura, 2006). Estas creencias ocupan un lugar central entre los pensamientos referidos a uno mismo, que afectan la acción y el funcionamiento humano (Bandura, 2012; Olaz y Pérez, 2012), a través de elementos cognitivos, motivacionales, afectivos y procesos de toma de decisiones. La teoría social cognitiva postula que altos niveles de autoeficacia para conductas específicas determinan una mayor persistencia en medio de las dificultades, aumentan la intencionalidad, la planificación a largo plazo, y promueven la autorregulación y las acciones de autocorrección (Bandura, 2012).

La autoeficacia no es un rasgo global, sino un conjunto de creencias ligadas a ámbitos de desempeño diferenciados (Bandura, 2006, 2012). Los sujetos no sólo emiten juicios de autoeficacia diferenciados y diferenciables para dominios diversos, sino que la autoeficacia puede y debe definirse con distintos niveles de especificidad en relación con un ámbito dado (e.j., área, tareas o problemas), de acuerdo con la conducta criterio de interés (Blanco, 2010). De esta manera, en el ámbito de la educación, la percepción de competencia de un estudiante y las creencias que él tenga para realizar una tarea, están directamente vinculadas al desempeño (Wang, Shannon \& Ross, 2013). Específicamente las calificaciones presen$\tan$ correlaciones de $r=0.35$ (Alegre, 2014) y $r=0.29$ con autoeficacia académica (Chemers, Hu, \& Garcia, 2001); $r=0.30$ con autoeficacia para el aprendizaje (Komarraju $\&$ Nadler, 2013); y $r=0.22$ con autoeficacia para el estudio (Putwain, Sander, \& Larkin, 2013).

La autoeficacia se asume como una aspecto clave en el proceso de control de la autorregulación (Zimmerman et al., 2005), siendo investigada en los estudiantes universitarios (Carot, Carranza, Olaz, \& Ponce, 2012; Guerreiro-Casanova, \& Polydoro, 2011). Se ha demostrado empíricamente que en contextos académicos es una de las variables motivacionales más importantes de la autorregulación del aprendizaje y una creencia clave que influye en el esfuerzo y la persistencia de los estudiantes en la realización de tareas (Huie et al., 2014). Desde este punto de vista, estudiantes con creencias de autoeficacia positiva hacia el aprendizaje son por lo general más motivados y obtienen mejor desempeño académico (Wang, Shannon, \& Ross, 2013). La investigación ha confirmado la validez predictiva de las creencias de autoeficacia en el desempeño, incluso cuando los posibles efectos del conocimiento previo y las habilidades cognitivas generales son controladas (Huie et al., 2014). Pese a la abundancia de estudios referidos a la relación entre autorregulación y autoeficacia (Demirören, Turan, \& Öztuna, 2016; Wäschle, Allgaier, Lachner, Fink, \& Nückles, 2014), faltan estudios específicos referentes a cada fase del proceso de autorregulación (Almeida \& Salcedo, 2013).

Definiremos la autoeficacia para la autorregulación de la disposición al estudio como las creencias que tienen los estudiantes acerca de sus capacidades para el logro de la autorregulación de las estrategias dirigidas a preparar 
la actividad de estudio (Pérez \& Delgado, 2006). Estas creencias impactan en el esfuerzo, persistencia y logro de las metas académicas (Bandura, 2012; Wang et al., 2013). La autoeficacia para la autorregulación de la disposición al estudio es una variable importante en la autorregulación del aprendizaje, ya que influye en la motivación académica de los alumnos, en las estrategias de autorregulación que utilizan en sus tareas y, en consecuencia, en el éxito académico que obtienen (Rosario et al., 2012).

Es necesario un instrumento específico para medir la autoeficacia para la autorregulación de la disposición al estudio, ya que si bien se cuenta con instrumentos para la medición de la autoeficacia académica (Barraza, 2010) y del estudio (Pérez \& Delgado, 2006), la construcción de escalas válidas de autoeficacia requiere determinantes sólidos que gobiernan el desempeño en un dominio específico de funcionamiento para realizar los logros deseados, tal como recomienda Bandura (2006).

El objetivo del trabajo es diseñar y estudiar el comportamiento psicométrico del Cuestionario de Autoeficacia para la Autorregulación de la Disposición al Estudio (CAPADE). Específicamente se busca determinar la confiabilidad del instrumento, así como recolectar indicadores de validez dimensional y convergente. Este instrumento de autoinforme fue diseñado en el marco del proyecto de investigación "Impacto de un programa de entrenamiento en estrategias y creencias para la disposición al estudio". Poseer un instrumento confiable y con indicadores de validez, permite tanto diagnosticar y establecer perfiles de alumnos con respecto a su autoeficacia para la autorregulación de la disposición al estudio, así como evaluar el éxito de intervenciones sobre esta dimensión.

\section{Método}

\section{Participantes}

La muestra del estudio estuvo compuesta por 695 estudiantes de Educación Superior. Participaron 15 programas de estudios de 5 Universidades de la Provincia de Concepción, Chile - Universidad de Concepción, Universidad Católica de la Santísima Concepción, Universidad Andrés Bello, Universidad Santo Tomás y Universidad del Desarrollo. Un 49.1\% de los participantes fueron mujeres $(n=341)$ y la edad promedio de los alumnos fue de 21.2 años $(D E=2.78)$. El muestreo de los participantes fue no probabilístico de conveniencia por grupos intactos, correspondientes a grupos de alumnos pertenecientes a una asignatura que fueron encuestados en sus horarios de clases.

Se dividió a la muestra total en dos partes. En la primera mitad se realizaron los análisis factoriales de carácter exploratorio $(n=347)$ y en la segunda mitad, se realizaron los análisis factoriales de tipo confirmatorio $(n=348)$. Se estratificaron las muestras por sexo, edad y universidad de pertenencia, debido a que fueron predictores estadísticamente significativos en los análisis de regresión ordinal realizados sobre cada ítem de la escala.

\section{Instrumento}

Cuestionario de Autoeficacia para la Autorregulación del Estudio. El Cuestionario de autoeficacia para la autorregulación de la disposición al estudio (CAPADE) fue diseñado por los autores de esta investigación. Es un instrumento de autoinforme, compuesto por nueve ítems, que tributan a una dimensión principal y tres secundarias definidas teóricamente correspondientes a 1. establecimiento de objetivos de académicos 2. gestión del tiempo académico y 3 . organización de recursos materiales y ambientales; cada una de estas tres dimensiones consta de tres ítems.

El participante debe responder a la consigna "creo que puedo utilizar la siguiente estrategia" para cada una de las estrategias de autorregulación del estudio, en una escala tipo Likert de 1 a 5 , donde 1 es "nada seguro" y 5 , "muy seguro". En la Tabla 1 se presentan los ítems del instrumento.

Tabla 1

Ítems del Instrumento

\begin{tabular}{cl}
\hline $\mathrm{N}^{\circ}$ & \multicolumn{1}{c}{ Ítem } \\
\hline 1 & Fijarme objetivos académicos (por ejemplo, obtener una nota elevada). \\
2 & Hacer una lista de tareas académicas por hacer. \\
3 & Tener el material necesario antes de empezar a estudiar. \\
4 & Gestionar el tiempo para actividades académicas, considerando el resto de actividades personales. \\
5 & Buscar un lugar óptimo para estudiar. \\
6 & Cumplir con el horario de estudio planificado. \\
7 & Dividir un objetivo complejo en metas más pequeñas y manejables. \\
8 & Organizar mi lugar de estudio. \\
9 & Revisar mis objetivos para hacer cambios si es necesario.
\end{tabular}

Nota. Estructura teórica. Factor 1. Establecimiento de Objetivos (ítems: 1-7-9); Factor 2. Gestión del tiempo académico (ítems2-4-6); Factor 3. Organización de recursos materiales y ambientales (ítems: 3-5-8). 


\section{Procedimiento}

Para la construcción del CAPADE, se adaptaron cuatro ítems del Inventario de Autoeficacia para el Estudio (Pérez \& Delgado, 2006) relacionados con el establecimiento de objetivos y se agregaron cinco ítems de elaboración, concordantes con la teoría de la autorregulación (Zimmerman, 2008). Específicamente se consideraron aspectos de la disposición (Pérez et al., 2011) y los lineamientos para construir escalas de Bandura (2012).

Para obtener evidencias de validez de contenido y de proceso, se realizó revisión por jueces expertos, y entrevista cognitiva, respectivamente. La validación de jueves expertos se realizó con seis psicólogos con estudios de postgrado, específicamente cinco jueces nacionales y 1 juez internacional. A cada juez se le envió un formulario en el cual se le presentó el instrumento y se le preguntó por la claridad, pertinencia y suficiencia de los ítems con un criterio dicotómico (cumple, no cumple), para dar cuenta del constructo autoeficacia para la autorregulación de la disposición al estudio. Finalmente, se solicitó realizar comentarios. Se incluyeron todos los ítems ya que hubo acuerdo en que todos los ítems eran pertinentes por al menos cinco jueces. Se mejoró la redacción en los ítems con problemas de claridad, declarados por más de un juez. El cambio más relevante, sugerido por todos los jueces, fue adaptar el vocabulario de los ítems del IDAPE (Pérez \& Delgado, 2006) a la cultura chilena.

Una vez modificado el instrumento tras el juicio de expertos, se realizaron seis entrevistas cognitivas con estudiantes de pregrado de Ingeniería, para detectar problemas de comprensión en los ítems. La entrevista cognitiva comenzaba con la explicación del objetivo de la actividad al participante; se le pedía que contestase el instrumento, anotando en un costado de cada ítem o expresando en voz alta (a) con sus propias palabras a qué refiere cada ítem (b) cualquier comentario o sugerencia que le surgiese en el proceso de respuesta. La entrevista cognitiva mostró una adecuada comprensión de los ítems por los estudiantes.

La aplicación del instrumento se realizó entre los meses de marzo y mayo de 2016 . Se contactó a diferentes autoridades de las cinco universidades, quienes indicaron que cursos podían ser partícipes de la investigación. Se contactó a los docentes de estos cursos, para coordinar la forma de aplicación de los instrumentos; los docentes informaron de la investigación con antelación a sus alumnos. En el día planificado para cada aplicación, se entregaron los consentimientos informados a los alumnos y se entregó el cuestionario a quienes aceptaban participar. El tiempo de aplicación del cuestionario no superó los 10 minutos por persona. Esta investigación fue aprobada por el Comité Ética y Bioética del Departamento de Psicología de la Universidad de Concepción, Chile.

\section{Análisis de datos}

Se dividió a la muestra total en dos partes igual. La primera mitad se realizaron análisis factoriales de carácter exploratorio $(n=347)$ y en la segunda mitad, se realizaron análisis factoriales de tipo confirmatorio $(n=348)$. Las dos submuestras se generaron estratificando por sexo, edad y universidad de pertenencia, debido a que fueron predictores estadísticamente significativos en análisis de regresión ordinal realizados sobre cada ítem.

Sobre la muestra para el análisis exploratorio, se decidió usar la matriz de correlaciones policóricas, por ser los ítems medidos a nivel ordinal y porque la asimetría y curtosis de tres de los nueve ítems superan el valor absoluto 1 (matriz de correlaciones policóricas disponible en anexo 1). Tras determinar la adecuación de la matriz de correlaciones para el análisis factorial utilizando la prueba de Bartlett y el criterio $\mathrm{KMO}$, se utiliza el método paralelo de Horn para determinar el número de factores, complementado con los criterios MAP de Velicer y VSS.

Como estrategia general para analizar la calidad de los análisis factoriales exploratorios, se considera que se pueden usar SEM - tradicionalmente asociado a los análisis factoriales confirmatorios -, en el enfoque denominado SEM-E (Marsh, Morin, Parker, \& Kaur, 2014). En nuestro caso, la estructura bifactorial que resulta del análisis resulta especialmente apropiada para un análisis de SEM-E bifactorial (Morin, Arens, \& Marsh, 2016). $\mathrm{Al}$ definir los ítems asociados a cada factor específico en los distintos análisis exploratorios, se realiza un análisis SEM, en el cual de forma preliminar se fijan a cero las cargas factoriales de los ítems que no se asocian a un factor específico, corrigiendo cualquier error de especificación en esta etapa; se puede considerar éste método como un análisis factorial confirmatorio preliminar, previo al análisis factorial confirmatorio definitivo en la muestra respectiva. En los análisis SEM, se utilizó el estimador WLSMV, apropiado para ítems de nivel de medición ordinal, y como indicadores de ajuste de los datos al modelo se usaron chi-cuadrado, CFI, TLI y RMSEA.

Tras el análisis de los índices de modificación del SEM de la solución unidimensional inicial, se consideró establecer una solución bifactorial basada en la estructura teórica, la cual se analizó mediante rotación de Procrustes, así como un tercer modelo bifactorial empírico basado en la transformación de Schmid Leiman. Los tres modelos (unidimensional, bifactorial teórico y bifactorial empírico) que fueron antes analizados en la muestra exploratoria, fueron posteriormente probados en la muestra confirmatoria usando SEM, constituyéndose así en un análisis factorial confirmatorio tradicional.

Finalmente, se evaluó la validez convergente del test al correlacionar la escala general y las subescalas con la nota obtenida del semestre anterior reportada por los 
alumnos, estandarizada por universidad y por carrera, en la muestra confirmatoria.

Como software de análisis se utilizó $\mathrm{R}$, versión 3 . Para los análisis exploratorios usamos el paquete psych (Revelle, 2017). Para los análisis con SEM se utilizó el paquete lavaan (Rosseel, 2012).

\section{Resultados}

Al analizar las diferencias en los ítems entre las muestras del análisis exploratorio y confirmatorio no superan los 0.17 en la media y 0.06 en la desviación estándar en los ítems. Los ítems 3, 5, 8 presentan asimetría inferiores a -1 en ambas muestras, en tanto que los ítems 5 y 8 presentan curtosis superiores a 1 en ambas muestras. El test multivariado de Mardia muestra que tanto la asimetría como la curtosis de ambas muestras superan la de una distribución multivariada normal $(p<0.001)$. Por tanto, se decidió usar la matriz de correlaciones policóricas para todos los análisis posteriores. El resultado del test de Bartlett indicó que la matriz de correlaciones policóricas no proviene de una matriz identidad, $\chi^{2}(36)=1458.7, p<0.001$. Además el índice $\mathrm{KMO}=0.83$ indicó que la matriz de correlaciones es apta para el análisis factorial.

El análisis paralelo de Horn sobre 500 remuestras correspondientes a permutaciones de los datos en bruto utilizando correlaciones policóricas mostró que la solución preferida es de un factor, al igual que el criterio MAP de Velicer y el VSS de complejidad 1. La solución de 1 factor obtenida con mínimos cuadrados predice un 44\% de la varianza y todos los ítems presentan una carga superior a 0.5. El alfa de Cronbach de la escala formada a partir de la totalidad de los ítems es 0.84 . El modelo SEM para el modelo unidimensional no se ajusta de forma absoluta a los datos, $\chi^{2}(27)=311,4, p<0.001$. Los indicadores de ajuste relativo muestran que la solución no es buena. Los indicadores $\mathrm{CFI}=0,88$ y $\mathrm{TLI}=0.84$, se encuentran bajo el mínimo de 0,95 esperado y RMSEA $=0.164, p<0.001$ y está sobre el máximo de 0.05 .

Al revisar los índices de modificación se encontró que los tres principales índices de modificación son correlaciones entre los residuos de 5 y $8(I M=155.5)$, de los ítems 7 y $9(I M=27,7)$ y de los ítems 2 y $4(I M=23,8)$. Estos tres pares corresponden a ítems que en su redacción aluden a establecimiento de objetivos, gestión del tiempo académico y organización de recursos respectivamente. Estas dimensiones fueron las que originalmente se plantearon para el instrumento. Por lo tanto, se propuso una estructura bifactorial donde todos los ítems tributaron al factor principal y a la vez a un factor secundario, de acuerdo a la propuesta teórica. El alfa de Cronbach para cada una de las dimensiones son para establecimiento de objetivos académicos $\alpha=0.69$, gestión del tiempo académico $\alpha=0.75$, y para organización de recursos materiales y ambientales $\alpha=0.76$.
El modelo SEM que consideró un factor general y tres factores de acuerdo a la teoría, no logra ajustar debido a que se encontró una solución inadmisible donde I4 presentó una carga factorial superior a 1. Para solucionar esto, se fijaron como cargas iguales las cargas en el factor 2 para los ítems 2,4 y 6 , lo que generó una solución admisible. El indicador de ajuste absoluto indicó que los datos aún no se ajustaban al modelo, $\chi^{2}(20)=111.105, p<0.001$. Si bien los indicadores $\mathrm{CFI}=0,96$ y $\mathrm{TLI}=0.93$, se encuentran sobre el mínimo y el otro bajo el mínimo de 0,95 esperado, el RMSEA $=0.12, p<0.001$ está sobre el máximo de 0.05 . Por lo tanto, si bien este modelo es mejor que el unidimensional puro, no logró ser adecuado.

Para encontrar una solución bifactorial óptima, se realizó una transformación de Schmid Leiman sobre la matriz de correlaciones policóricas considerando tres factores secundarios. El omega total de la escala completa, que indica la proporción de varianza común, es de $\omega_{\mathrm{t}}=.88$, y el omega jerárquico, que indica la proporción de varianza del factor común, es $\omega_{\mathrm{j}}=.68$. Un primer factor se forma por los ítems 1, 6, 7 y 9 , que corresponden a establecimiento de objetivos $(\alpha=.76$, $\left.\omega_{\mathrm{t}}=.76, \omega_{\mathrm{j}}=.51\right)$. El segundo factor está compuesto por los ítems 2, 3, 4 y 6, que corresponden a gestión del tiempo académico $\left(\alpha=.76, \omega_{\mathrm{t}}=.74, \omega_{\mathrm{j}}=.48\right)$. El tercer factor está compuesto por los ítems 3,5 y 8 , que corresponden a organización de recursos materiales y ambientales $\left(\alpha=.76, \omega_{\mathrm{t}}=.79, \omega_{\mathrm{j}}=.37\right)$.

En el análisis se observaron dos ítems con cargas cruzadas entre sus subfactores ( 3 y 6 ), que son justificables teóricamente. El ítem 3 "tener el material necesario antes de empezar a estudiar”, combina la organización de recursos materiales y ambientales con la gestión del tiempo académico. El ítem 6 "cumplir con el horario de estudio planificado", a su vez, une la gestión del tiempo académico, con el establecimiento de objetivos académicos.

La solución de SEM para el modelo bifactorial empírico con esta estructura indica que los datos no se ajustan al modelo, $\chi^{2}(16)=46.495, p<0.001$. Los indicadores de ajuste relativo muestran que la solución es adecuada. Los indicadores CFI $=0,99$ y TLI $=0.97$, se encuentran sobre el mínimo de 0,95 esperado y RMSEA $=0.074, p=0.51$, si bien está sobre 0.05 , no es significativo.

Las distintas cargas factoriales para las soluciones exploratorias se presentan en la tabla 2. Se puede observar que los ítems 5, 6, 7, 8 y 9 en la solución bifactorial teórica obtenida mediante rotación de Procrustes presentan cargas mayores en el subfactor que en el factor general. En cambio, en la solución empírica solamente los ítems 5 y 8 presentan mayor carga factorial en el subfactor que en el factor general, lo que da cuenta de un modelo fundamentalmente unidimensional. 
Tabla 2

Cargas Factoriales y Descriptivos para Modelos Unidimensional y Bifactorial en Muestra Exploratoria

\begin{tabular}{|c|c|c|c|c|c|c|c|c|c|c|}
\hline \multirow{2}{*}{ Ítem } & \multirow{2}{*}{ Dimensión } & \multirow{2}{*}{$\begin{array}{l}\text { UD } \\
\text { FG }\end{array}$} & \multicolumn{4}{|c|}{ Bifactorial-teórico } & \multicolumn{4}{|c|}{ Bifactorial-empírico } \\
\hline & & & FG & F1 & F2 & F3 & FG & F1 & F2 & F3 \\
\hline 1 & EO & 0,55 & 0,53 & 0,00 & 0,31 & $-0,05$ & 0,44 & 0,20 & 0,16 & 0,04 \\
\hline 2 & GTA & 0,68 & 0,76 & $-0,07$ & $-0,01$ & 0,24 & 0,57 & 0,04 & 0,45 & 0,01 \\
\hline 3 & ORMA & 0,68 & 0,74 & 0,28 & $-0,03$ & $-0,04$ & 0,50 & $-0,06$ & 0,27 & 0,29 \\
\hline 4 & GTA & 0,71 & 0,69 & 0,03 & $-0,13$ & 0,43 & 0,59 & 0,02 & 0,49 & 0,01 \\
\hline 5 & ORMA & 0,65 & 0,42 & 0,83 & $-0,12$ & 0,11 & 0,51 & $-0,03$ & 0,03 & 0,55 \\
\hline 6 & GTA & 0,72 & 0,44 & 0,02 & 0,17 & 0,72 & 0,62 & 0,34 & 0,23 & $-0,02$ \\
\hline 7 & EO & 0,64 & 0,41 & 0,03 & 0,48 & 0,16 & 0,55 & 0,45 & 0,05 & 0,01 \\
\hline 8 & ORMA & 0,69 & 0,52 & 0,64 & 0,18 & $-0,13$ & 0,60 & 0,06 & $-0,02$ & 0,61 \\
\hline 9 & EO & 0,64 & 0,40 & 0,02 & 0,72 & 0,03 & 0,57 & 0,53 & $-0,05$ & 0,05 \\
\hline \multicolumn{2}{|c|}{ V. explicada } & $44 \%$ & $31,7 \%$ & $13,0 \%$ & $10,5 \%$ & $9,1 \%$ & $30,6 \%$ & $7,2 \%$ & $6,7 \%$ & $8,5 \%$ \\
\hline \multicolumn{2}{|c|}{$\mathrm{M}$} & 3.87 & 3.87 & 4.30 & 3.65 & 3.66 & 3.87 & 3.62 & 3.85 & 4.30 \\
\hline \multicolumn{2}{|l|}{$D E$} & 0.65 & 0.65 & 0.69 & 0.80 & 0.87 & 0.65 & 0.78 & 0.75 & 0.69 \\
\hline
\end{tabular}

Nota. UD: Modelo Unidimensional. EO: Establecimiento de Objetivos. GTA: Gestión del tiempo académico. ORMA: Organización de los recursos materiales y ambientales. En negrita cargas factoriales sobre 0.2. Media y Desviación Estándar corresponde a la escala formada por todos los ítems que presentan carga factorial superior a 0.3 en el factor respectivo.

\section{Análisis Factorial Confirmatorio}

En la tabla 3 se observan los resultados del análisis factorial confirmatorio sobre la muestra de prueba de 347 sujetos. Ninguna de las soluciones se ajustó en forma absoluta a los datos usando $\chi^{2}, p<0.001$. El modelo unidimensional no logró presentar indicadores adecuados de ajuste, ya que el $\chi^{2}$ normalizado es superior a 9, presenta CFI y TLI menores a 0,95 y el RMSEA es elevado, aproximadamente 0,155 . El modelo bifactorial teórico, si bien presentó un menor ajuste con $\chi^{2}$ normalizado de 5,4 y CFI $=0.96$, aun presenta un RMSEA alto, de 0.113. El mejor modelo disponible es el bifactorial empírico, con $\chi^{2}$ normalizado igual a 2.1, valores altos de CFI $=0.99$ y TLI $=0.98$. Su RMSEA $=0.055$, si bien no óptimo por ser mayor a 0.05 , no es significativo.

Tabla 3

Análisis Factorial Confirmatorio para Modelo Unidimensional, Bifactorial Teórico y Bifactorial Empírico

\begin{tabular}{lccccc}
\hline \multicolumn{1}{c}{ Modelo } & $\chi^{2}$ & $\chi^{2} / g l$ & CFI & TLI & RMSEA \\
\hline Unidimensional & $\chi^{2}(27)=251.7, p<0.001$. & 9,3 & 0,90 & 0,87 & $0,155, p<0,001$ \\
Bifactorial Teórico & $\chi^{2}(20)=107.85, p<0.001$. & 5,4 & 0.96 & 0,93 & $0,113, p<0,001$ \\
Bifactorial Empírico & $\chi^{2}(16)=32.85, p=0.008$ & 2,1 & 0,99 & 0,98 & $0,055, p=0,343$ \\
\hline
\end{tabular}

\section{Relación entre Calificaciones y Autoeficacia para la Autorregulación de la Disposición al Estudio}

$\mathrm{Al}$ analizar gráficamente la relación entre las puntaciones de las escalas del modelo empírico del CAPADE y las calificaciones autorreportadas, estas resultan ser aproximadamente lineales, por lo que es apropiado utilizar la correlación momento-producto de Pearson. La relación entre las calificaciones y la escala general es de $r=0.13, p=0.02$; con la subescala 1 Establecimiento de Objetivos Académicos es de $r=0.08, p=0.17$; con la subescala 2 Gestión del Tempo Académico es $r=0.17, p<0.01$; y la subescala 3 Organización de Recursos Materiales y Ambientales es de $r=0.11, p=0.04$.

\section{Discusión}

El objetivo del trabajo fue diseñar y determinar el comportamiento psicométrico del instrumento (CAPADE), en estudiantes universitarios chilenos. En el diseño del instrumento se adaptaron 4 ítems del Inventario de Autoeficacia para el estudio (Pérez \& Delgado, 2006) y se diseñaron otros 5 en pertinencia con la teoría. El instrumento fue presentado a jueces expertos y se aplicó una entrevista cognitiva a los estudiantes. Este proceso mostró suficiencia, claridad y pertinencia de los ítems por los jueces expertos y comprensión por parte de los estudiantes de la escala diseñada. 
$\mathrm{Al}$ analizar las propiedades psicométricas se encontró que el instrumento presenta una dimensión principal $\mathrm{y}$ tres subdimensiones secundarias correspondientes a las dimensiones teóricas: establecimiento de objetivos académicos, gestión del tiempo académico y organización de recursos materiales y ambientales. Estas subdimensiones presentan ítems con cargas cruzadas justificables desde un punto de vista lógico. Cada una de las escalas formadas por la dimensión general y las subdimensiones presentan alfa de Cronbach adecuado superiores a 0.7. Además la solución presenta buenos indicadores de ajuste usando en el análisis factorial confirmatorio y muestra correlaciones leves, pero estadísticamente significativas, con las calificaciones del semestre anterior reportadas por los estudiantes.

La estructura bifactorial del instrumento puede ser explicada atendiendo al carácter dual del constructo medido, la autoeficacia sobre las estrategias de disposición al estudio. Se reconoce que la autorregulación del estudio, involucra una variedad de estrategias que son utilizadas de acuerdo a la tarea de aprendizaje y las características del alumno. En particular la disposición al estudio requiere estrategias relacionadas al establecimiento de objetivos, gestión de recursos materiales y ambientales y gestión del tiempo, las cuales se ven reflejadas como subfactores del instrumento. Sin embargo, al ser la variable medida la creencia sobre la capacidad de realizar acciones de preparación sobre el estudio, el éxito del desempeño pasado afecta la autoeficacia para desplegar el conjunto de estrategias, lo que explica el fuerte factor general (Kitsantas, Winsler, \& Huie, 2008)

La complejidad de la estructura del instrumento en términos de los factores secundarios donde las estrategias se relacionan con un segundo factor, se explica por lo complejo de los modelos de autorregulación y el carácter multidimensional de las estrategias de autorregulación (disposicional, cognitivo, metacognitivo, motivacional, de gestión de recursos, de control, y gestión del esfuerzo, etc.). Se enfatiza la concepción multidimensional de la autorregulación a la hora de explicar el comportamiento del estudiante durante una tarea de aprendizaje (Dinsmore, Alexander, \& Loughlin, 2008).

Las correlaciones de las subdimensiones del instrumento con las calificaciones se encuentran en el rango entre $r=0.08$, y $r=0.1$, en tanto que la correlación de la escala general con el calificaciones es de $r=0.13$. Estas correlaciones son menores a las reportadas en la literatura entre las calificaciones y autoeficacia académica (Alegre, 2014), autoeficacia del aprendizaje (Komarraju, \& Nadler, 2013) y autoeficacia para el estudio (Putwain et al.,2013), que oscilan entre $r=0.22$ y $r=0.35$. Esto se puede explicar por dos motivos: (a) la nota es autorreportada, por lo tanto presenta una menor precisión que una nota obtenida a partir de un registro académico, y (b) que el constructo medido en la escala CAPADE es parte de un proceso muy específico de la autorregulación del estudio, a diferencia de las escalas de autoeficacia académica general y autoeficacia del aprendizaje que al medir conductas más generales, comprende también las actividades de evaluación de las cuales dependen directamente las notas.

En conclusión, el CAPADE se considera un instrumento fiable y válido, de fácil y rápida aplicación, para la medición de la autoeficacia para la autorregulación de la disposición al estudio en estudiantes universitarios. Puede ser útil para la evaluación del impacto de programas de intervención de autorregulación y para la investigación de la relación de autoeficacia y otras variables académicas.

La principal fortaleza del instrumento es su estructura bifactorial, que brinda tanto una medición única de la autoeficacia para la autorregulación de la disposición al estudio, como subfactores que permiten medir aspectos específicos de este proceso. Además, este instrumento es pertinente para distintos grupos de estudiantes, ya que la muestra estuvo compuesta por un alto número de estudiantes, correspondiente a diversas carreras de distintas áreas del conocimiento (Educación, Salud, Ingeniería y Geología), universidades y niveles de estudio de Educación Superior. Como limitaciones del estudio, las calificaciones son autorreportadas desconociéndose una nota del registro académico del final del semestre para haber realizado un análisis predictivo de las calificaciones en función de la autoeficacia.

Un posible paso a seguir sería analizar la relación del instrumento con calificaciones formales obtenidas del registro académico de los estudiantes, y no con notas autorreportadas como fue en este estudio. Esto permitirá obtener una medida más precisa de la autoeficacia para autorregular la disposición al estudio y el desempeño académico de los estudiantes.

\section{Referencias}

Alegre, A. (2014). Autoeficacia académica, autorregulación del aprendizaje y rendimiento académico en estudiantes universitarios iniciales. Propósitos y Representaciones, 2(1), 79-120. doi: 10.20511/pyr2014.v2n1.54

Almeida, B., \& Salcedo, I. (2013). La autorregulación en la actividad de estudio: Procedimientos que pueden emplearse para su desarrollo en la clase de matemática. Revista Científica Pedagógica Atenas, 4(21), 17-33. Recuperado de http://atenas.mes.edu.cu/index.php/atenas/ article/view/21/pdf 
Bandura, A. (2006). Guide for constructing self-efficacy scales. En F. Pajares \& T. Urdan (Eds.), Self-efficacy Beliefs of Adolescents (pp. 307-337). Greenwich, CT: Information Age Publishing.

Bandura, A. (2012). On the functional properties of perceived self-efficacy revisited. Journal of Management, 38(1), 9-44. doi: $10.1177 / 0149206311410606$

Barraza, A. (2010). Validación del inventario de expectativas de autoeficacia académica en tres muestras secuenciales e independientes. CPU-e, Revista de Investigación Educativa, (10), 1-30. Recuperado de http://revistas.uv.mx/index.php/cpue/article/view/65/881

Blanco, A. (2010). Creencias de autoeficacia de estudiantes universitarios: Un estudio empírico sobre la especificidad del constructo. Revista Electrónica de Investigación y Evaluación Educativa, 16(1), 1-28. Recuperado de http://www.uv.es/RELIEVE/v16n1/RELIEVEv16n1_2.pdf

Carot, J., Carranza, E., Olaz, F., \& Ponce, L. (2012). Autoeficacia para la Investigación en Estudiantes Universitarios. Revista Tesis, 2 (1), 61-77. Recuperado de https://revistas.unc.edu.ar/index.php/tesis/article/view/2878

Chemers, M., Hu, L., \& Garcia, B. (2001). Academic self-efficacy and first year college student performance and adjustment. Journal of Educational Psychology, 93(1), 55-64. doi: 10.1037//0022-0663.93.1.55

Demirören, M., Turan, S., \& Öztuna, D. (2016). Medical students' self-efficacy in problem-based learning and its relationship with selfregulated learning. Medical education online, 21(1), 1-9. doi: 10.3402/meo.v21.30049

Dinsmore, D., Alexander, P., \& Loughlin, S. (2008). Focusing the conceptual lens on metacognition, self-regulation, and self-regulated learning. Educational Psychology Review, 20(4), 391-409. doi: 10.1007/s10648-008-9083-6

Elvira-Valdés, M., \& Pujol, L. (2014). Variables cognitivas e ingreso universitario: Predictores del rendimiento académico. Universitas Psychologycal, 13(4), 1557-1567. doi: 10.11144/Javeriana.UPSY13-4.vciu

Guerreiro-Casanova, D., \& Polydoro, S. (2011). Autoeficácia e integração ao ensino superior: Um estudo com estudantes de primeiro ano. Psicologia: teoria e prática, 13(1), 75-88. Recuperado de http://pepsic.bvsalud.org/scielo.php?script=sci_arttext\&pid $=$ S1516-36872011000100006

Huie, F., Winsler, A., \& Kitsantas, A. (2014). Employment and first-year college achievement: The role of self-regulation and motivation. Journal of Education and Work, 27(1), 110-135. doi: 10.1080/13639080.2012.718746

Kitsantas, A., Winsler, A., \& Huie, F. (2008). Self-regulation and ability predictors of academic success during college: A predictive validity study. Journal of Advanced Academics, 20(1), 42-68. doi: 10.4219/jaa-2008-867

Komarraju, M., \& Nadler, D. (2013). Self-efficacy and academic achievement: Why do implicit beliefs, goals, and effort regulation matter? Learning and Individual Differences, 25, 67-72. doi: 10.1016/j.lindif.2013.01.005

Kornell, N., \& Bjork, R. (2007). The promise and perils of self-regulated study. Psychonomic Bulletin \& Review, 14(2), 219-224. doi: 10.3758/ BF03194055

Marsh, H., Morin, A., Parker, P., \& Kaur, G. (2014). Exploratory structural equation modeling: An integration of the best features of exploratory and confirmatory factor analysis. Annual Review of Clinical Psychology, 10(1), 85-110. doi: 10.1146/annurev-clinpsy-032813-153700

Morin, A., Arens, A., \& Marsh, H. (2016). A bifactor exploratory structural equation modeling framework for the identification of distinct sources of construct-relevant psychometric multidimensionality. Structural Equation Modeling: A Multidisciplinary Journal, 23(1), 116139. doi: 10.1080/10705511.2014.961800

Olaz, F., \& Pérez, E. (2012). Creencias de Autoeficacia: líneas de investigación y desarrollo de escalas. Revista Tesis, 2(1), 157-170. Recuperado de https://revistas.unc.edu.ar/index.php/tesis/article/view/2881

Pérez, E., \& Delgado, M. (2006). Inventario de autoeficacia para el estudio: Desarrollo y validación inicial. Avaliação Psicológica, 5(2), 135-143. Recuperado de http://pepsic.bvsalud.org/pdf/avp/v5n2/v5n2a03.pdf

Pérez, M., Valenzuela, M., Díaz, A., González-Pienda, J., \& Núñez, J. (2011). Disposición y enfoques de aprendizaje en estudiantes universitarios de primer año. Universitas Psychologica, 10(2), 441-449. Recuperado de http://revistas.javeriana.edu.co/index.php/ revPsycho/article/view/691

Putwain, D., Sander, P., \& Larkin, D. (2013). Academic self-efficacy in study-related skills and behaviours: Relations with learning-related emotions and academic success. British Journal of Educational Psychology, 83(4), 633-650. doi: 10.1111/j.2044-8279.2012.02084

Revelle,W. (2017). Psych: Procedures for Psychological, Psychometric, and Personality Research. Recuperado de https://CRAN.R-project.org/ package $=$ psych *

Rosseel, Y. (2012). Lavaan: An R package for structural equation modeling. Journal of Statistical Software, 48(2), 1-36. doi: 10.18637/jss.v048.i02

Rosario, P., Lourenço, A., Paiva, M., Núñez, J., González-Pineda, J., \& Valle, A. (2012). Autoeficacia y utilidad percibida como condiciones necesarias para un aprendizaje académico autorregulado. Anales de Psicología, 28(1), 37-44. Recuperado de http://revistas.um.es/analesps/ article/viewFile/140502/126592

Rosário, P., Mourão, R., Núñez, C., González-Pienda, J., Solano, P., \& Valle, A. (2007). Eficacia de un programa instruccional para la mejora de procesos y estrategias de aprendizaje en la enseñanza superior. Psicothema, 2007, 19(3), 422-427

Soderstrom, N., \& Bjork, R. A. (2014). Testing facilitates the regulation of subsequent study time. Journal of Memory and Language, 73, 99-115. doi: $10.1016 /$ j.jml.2014.03.003

Wang, C., Shannon, D., \& Ross, M. (2013). Students' characteristics, self-regulated learning, technology self-efficacy, and course outcomes in online learning. Distance Education, 34(3), 302-323. doi: 10.1080/01587919.2013.835779

Wäschle, K., Allgaier, A., Lachner, A., Fink, S., \& Nückles, M. (2014). Procrastination and self-efficacy: Tracing vicious and virtuous circles in self-regulated learning. Learning and instruction, 29, 103-114. doi: 10.1016/j.learninstruc.2013.09.005

Yan, V., Thai, K., \& Bjork, R. A. (2014). Habits and beliefs that guide self-regulated learning: Do they vary with mindset? Journal of Applied Research in Memory and Cognition, 3(3), 140-152. doi:1 0.1016/j.jarmac.2014.04.003

Zimmerman, B. (2002). Becoming a self-regulated learner: An overview. Theory Into Practice, 41(2), 64-70. doi: 10.1207/s15430421tip4102_

Zimmerman, B. (2008). Investigating self-regulation and motivation: Historical background, methodological developments, and future prospects. American Educational Research Journal, 45(1), 166-183. doi: 10.3102/0002831207312909

Zimmerman, B., Kitsantas, A., \& Campillo, M. (2005). Evaluación de la autoeficacia regulatoria: una perspectiva social cognitiva. Revista Evaluar, 5, 1-21. Recuperado de https://revistas.unc.edu.ar/index.php/revaluar/article/view/537/477 
Apêndice

Correlaciones Policóricas entre Ítems

\begin{tabular}{|c|c|c|c|c|c|c|c|c|c|}
\hline & I. 62 & I.63 & I.64 & I.65 & I.66 & I.67 & I.68 & I.69 & I.70 \\
\hline I.62 & 1 & & & & & & & & \\
\hline I.63 & 0.4312 & 1 & & & & & & & \\
\hline I.64 & 0.4349 & 0.5422 & 1 & & & & & & \\
\hline I.65 & 0.3476 & 0.6178 & 0.5276 & 1 & & & & & \\
\hline I.66 & 0.2731 & 0.3292 & 0.5229 & 0.4172 & 1 & & & & \\
\hline I.67 & 0.3564 & 0.5014 & 0.3554 & 0.5908 & 0.4379 & 1 & & & \\
\hline I.68 & 0.3817 & 0.3718 & 0.3285 & 0.418 & 0.3254 & 0.5737 & 1 & & \\
\hline I.69 & 0.3336 & 0.3911 & 0.5548 & 0.4068 & 0.7449 & 0.3877 & 0.4234 & 1 & \\
\hline I.70 & 0.4254 & 0.3758 & 0.3247 & 0.3489 & 0.3282 & 0.5847 & 0.5855 & 0.4507 & 1 \\
\hline
\end{tabular}

\section{Sobre os autores}

Fabiola Sáez-Delgado é psicóloga, Doutora em Psicologia pela Universidad de Concepción.

Claudio Bustos-Navarrete é psicólogo, Mestre em Psicologia pela Universidad de Concepción.

Alejandro Díaz-Mujica é psicólogo, Doutor em Psicologia pela Universidad de Concepción. 